\title{
Quorum-Based Asynchronous Power-Saving Protocols for IEEE 802.11 Ad Hoc Networks
}

\author{
Jehn-Ruey Jiang*, Yu-Chee Tseng ${ }^{\dagger}$, Chih-Shun Hsu ${ }^{\ddagger}$ and Ten-Hwang Lai ${ }^{\S}$ \\ * Department of Information Management \\ Hsuan-Chuang University, Taiwan \\ ${ }^{\dagger}$ Department of Computer Science and Information Engineering \\ National Chiao-Tung University, Taiwan \\ ${ }^{\ddagger}$ Department of Computer Science and Information Engineering \\ National Central University, Taiwan \\ $\S$ Department of Computer and Information Science \\ The Ohio State University Columbus, OH 43210, USA
}

\begin{abstract}
This paper investigates the power mode management problem for an IEEE 802.11-based mobile ad hoc network (MANET) that allows mobile hosts to tune to the power-saving (PS) mode. There are two major issues that need to be addressed in this problem: (a) wakeup prediction and (b) neighbor discovery. The former is to deliver buffered packets to a PS host at the right time when its radio is turned on. The latter is to monitor the environment change under a mobile environment. One costly, and not scalable, solution is to time-synchronize all hosts. Another possibility is to design asynchronous protocols as proposed by Tseng et al. in [25]. In this paper, we adopt the latter approach and correlate this problem to the quorum system concept. We identify a rotation closure property for quorum systems. It is shown that any quorum system that satisfies this property can be translated to an asynchronous power-saving protocol for MANETs. Thus, the result bridges the classical quorum system design problem in the area of distributed systems to the power mode management problem in the area of mobile ad hoc networks. We derive a lower bound for quorum sizes for any quorum system that satisfies the rotation closure property. We identify a group of quorum systems that are optimal or near optimal in terms of quorum sizes, which can be translated to efficient asynchronous power-saving protocols. We also propose a new e-torus quorum system, which can be translated to an adaptive protocol that allows designers to trade hosts' neighbor sensibility for power efficiency. Simulation experiments are conducted to evaluate and compare the proposed protocols.
\end{abstract}

Keywords: IEEE 802.11, distributed system, mobile ad hoc network (MANET), power management, quorum system, wireless communication.

\section{Introduction}

The mobile ad hoc network (MANET) has attracted a lot of attention recently. A MANET consists of a set of mobile hosts, and does not have the support of any base station. Hosts may communicate in a multi-hop manner. Applications of MANETs include communications in battlefields, disaster rescue operations, and outdoor activities. 
Power saving is a critical issue for portable devices supported by batteries. Battery power is a limited resource, and it is expected that battery technology is not likely to progress as fast as computing and communication technologies do. Hence, how to save the energy consumption in a MANET, which is all supported by batteries, has been intensively studied recently (e.g., power control is studied in [8, 9, 17, 26, $28]$, power-aware routing in $[6,18,19,24]$, and low-power mode management in $[1,2,7,10,13,20,22,23$, $27,29])$.

This paper investigates the power mode management problem in an IEEE 802.11-based MANET, which is characterized by multi-hop communication, unpredictable mobility, and no plug-in power. IEEE 802.11 [11] has defined its power-saving (PS) mode for single-hop (fully connected) MANETs based on periodical transmissions of beacons. The protocol, when applied to a multi-hop MANET, may encounter several problems, including costly clock synchronization and even incorrect network partitioning [25].

There are two major issues that need to be addressed in the power mode management problem in a multi-hop MANET:

- Wakeup prediction: Since a host entering the PS mode will reduce its radio activity, other hosts who intend to send packets to the PS host need to know when the host will turn its radio on so as to correctly deliver packets to it at the right time.

- Neighbor discovery: Because hosts' transmission/reception activities are reduced under the PS mode, a host may take longer time, or may be even unable, to detect the arrival and departure of other hosts in its radio covered range. Thus, hosts may become less sensitive to neighborhood change. Neighbor discovery is essential for route discovery in a MANET. A host may incorrectly report that another host is unreachable if the route to this host has to go through some PS hosts that are not detectable by their neighbors on the path.

One possible solution to the above problems is to always time-synchronize all hosts. This approach is adopted by IEEE 802.11 under the ad hoc mode. However, 802.11 only considers single-hop MANETs. Time synchronization in a large-scale distributed environment is generally very costly. It is even infeasible in a mobile environment since communication delays are typically long and, worse, the MANET may be temporarily partitioned at any time, making time synchronization impossible. Another solution is to develop asynchronous power-saving protocols. This is first investigated in [25], where three solutions are proposed. Among them, the quorum-based protocol is probably the most interesting one. It has the merit of sending the fewest beacon signals (and is thus very energy-efficient). The central idea in the quorumbased protocol can be related to the grid quorum system [15]. This leads to a more general question: Can we apply other forms of quorum systems to this asynchronous power-saving problem? The result can potentially bridge the important quorum system concept in traditional distributed systems to the area of mobile computing, which may in turn generate more efficient asynchronous power-saving protocols. This 
Table 1: Power Consumption of the ORiNOCO IEEE 802.11b PC Gold Card (11 Mbps).

\begin{tabular}{|c|c|c|c|c|}
\hline Mode & PS (Doze) & Transmit & Receive & Monitor \\
\hline $\begin{array}{c}\text { Power } \\
\text { Consumed }\end{array}$ & $60 \mathrm{~mW}$ & $1400 \mathrm{~mW}$ & $950 \mathrm{~mW}$ & $805 \mathrm{~mW}$ \\
\hline
\end{tabular}

work does confirm such possibility.

In this paper, we correlate the asynchronous power-saving problem to the concept of quorum systems, which are widely used in the design of distributed systems $[3,12,14,15]$. A quorum system is a collection of sets such that the intersection of any two sets is always non-empty. Not all quorum systems are applicable to the power-saving problem. We identify a rotation closure property for quorum systems. It is shown that, through our mechanism, any quorum system satisfying this property can be translated to an asynchronous power-saving protocol for MANETs. We derive a lower bound for quorum sizes for any quorum system satisfying the rotation closure property. We identify a group of quorum systems that are optimal or near optimal in terms of quorum sizes (the grid quorum system [15], the torus quorum system [12], the cyclic quorum system [14], and the finite projective plane quorum system [15]), which can be translated to efficient asynchronous power-saving protocols. We also propose a new e-torus quorum system, which can be translated to an adaptive protocol that allows designers to trade hosts' neighbor sensibility for power efficiency. A host can dynamically adjust its beacon rate according to its mobility. Simulation experiments are conducted to evaluate and compare the proposed protocols in terms of the survival ratio, the route establishment probability, and the power efficiency.

The rest of this paper is organized as follows. Preliminaries are given in Section 2. Section 3 introduces the rotation closure property. Section 4 shows several quorum systems that satisfy this property. Section 5 presents our adaptive power-saving protocol. Simulation results are presented in Section 6. Conclusions are drawn in Section 7.

\section{Preliminaries}

\section{$2.1 \quad$ Power-Saving Modes in IEEE 802.11}

IEEE 802.11 supports two power modes: active and power-saving (PS). Under the PS mode, a host can reduce its radio activity by only monitoring some periodical signals (such as beacons) in the network. Tuning a host to the PS mode can save a lot of energy. For example, Table 1 summarizes the power consumption of ORiNOCO IEEE 802.11b PC Gold Card [21]. However, PS mode should be used cautiously so that the network throughput and delay do not get hurt.

Under the ad hoc mode, IEEE 802.11 divides the time axis into equal-length beacon intervals, each of which starts with an ATIM (Ad hoc Traffic Indication Map) window. The ATIM window is relatively small compared to the beacon interval. PS hosts must remain active during the ATIM window so as to be 


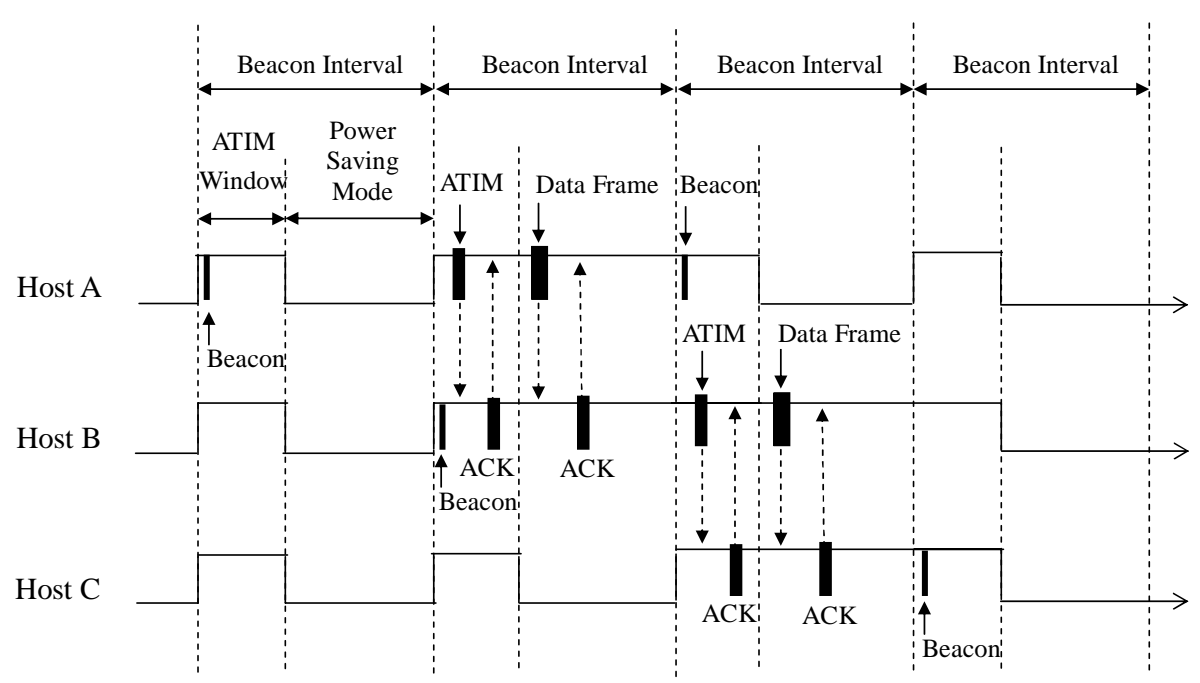

Figure 1: Transmission scenarios for PS hosts in a single-hop 802.11 MANET.

notified by those intending senders, and may go to doze in the rest of the beacon interval if no one intends to send packets to it. It is assumed that the ad hoc network is fully connected, so time synchronization is not an issue. In the beginning of a beacon interval, each mobile host will contend to send a beacon frame. Any successful beacon serves the purpose of synchronizing mobile hosts' clocks as well as inhibiting other hosts from sending their beacons. To avoid collisions, each beacon is led by a random backoff between 0 and $2 \times C W_{\min }-1$ slots.

After the beacon, a host with buffered packets can send a direct ATIM frame to each of its intended receivers in the PS mode. ATIMs are transmitted by contention in accordance with the DCF (Distributed Coordination Function) access procedure. A receiver, on hearing the ATIM, should reply an ACK and remain active. After the ATIM window, hosts having neither packets to send nor packets to receive can go back to the PS mode to save energy. The buffered unicast packets are then sent based on the DCF access procedure after the ATIM window. If the sender doesn't receive an ACK, it should retry in the next ATIM window. If a mobile host is unable to transmit its ATIM frame in the current ATIM window or has extra buffered packets, it should retransmit ATIMs in the next ATIM window. To protect PS hosts, only RTS, CTS, ACK, Beacon, and ATIM frames can be transmitted during the ATIM window. An example is illustrated in Fig. 1.

\subsection{Review: A Quorum-Based PS Protocol}

IEEE 802.11 only considers single-hop MANETs. For multi-hop MANETs, the following two issues have to be addressed: wakeup prediction and neighbor discovery. In [25], three solutions are proposed to solve these problems: the dominating-awake-interval, the periodically-fully-awake-interval, and the quorumbased protocols. Among them, the quorum-based one has the merit of sending the fewest beacon signals. Below, we briefly review the quorum-based protocol proposed in [25]. Still, the time axis is divided evenly 


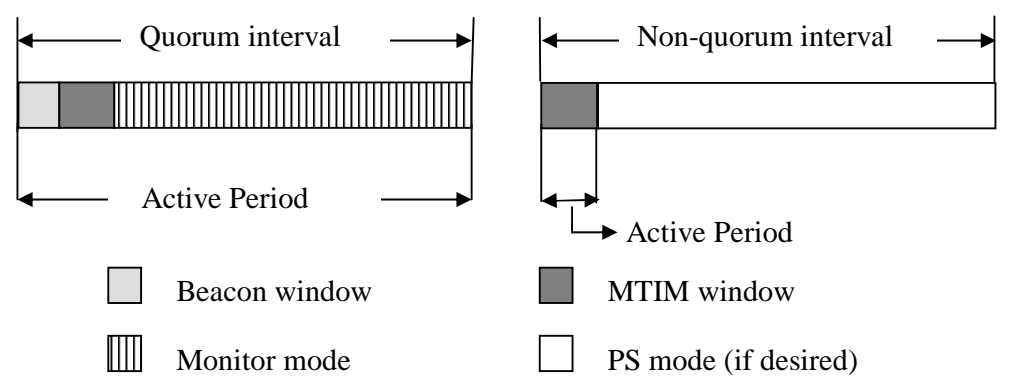

Figure 2: Structures of quorum intervals and non-quorum intervals.

into beacon intervals. Hosts can be arbitrarily asynchronous in their clocks. Beacon intervals are classified into two types (refer to Fig. 2):

- Quorum interval: It starts with a beacon window followed by a MTIM window. After the MTIM window, the host remains active (in monitor mode) for the rest of the beacon interval.

- Non-quorum interval: It starts with a MTIM window. After the MTIM window, the host may go to the PS mode if it has no packets to send or receive.

Similar to IEEE 802.11, the beacon window is for hosts to compete sending their beacons. The MTIM window is similar to the ATIM window - a host with buffered packets can compete to send notifications to intended receivers in the PS mode to wake them up. It is named so to reflect that it is used for multi-hop ad hoc networks. We assume that beacon windows are not longer than MTIM windows (the assumption is practical considering these two window's functionality; the assumption will also be used in our later proofs). With these definitions, we say that a PS host is active when it is currently in a beacon window, a MTIM window, or in a quorum interval.

In [25], it is proposed that each host divides its beacon intervals into groups such that each group consists of $n$ consecutive intervals. Each group is organized as an $\sqrt{n} \times \sqrt{n}$ array in a row-major manner. The host then picks intervals along an arbitrary row and an arbitrary column from the array as quorum intervals, and the remaining intervals as non-quorum intervals. Thus, there are $2 \sqrt{n}-1$ quorum intervals. It is shown that no matter how asynchronous hosts' clocks are, a PS host always has two or more beacon windows that are fully covered by another PS host's active period in every $n$ consecutive beacon intervals. Intuitively, this implies that two hosts can discover each other at least twice in every $n$ consecutive beacon intervals, if their beacon frames do not encounter collisions during transmission ${ }^{1}$. Thus, the neighbor discovery problem is resolved. Further, by carrying clock information in beacon frames, the wake-up prediction problem is also solved.

Fig. 3 shows an example with $n=16$. Host $A$ picks intervals along the first row and the second column as its beacon intervals. Host $B$, which does not coordinate with $A$, picks the third row and the third

\footnotetext{
${ }^{1}$ Collision is inevitable in any kind of contention-based MAC protocols.
} 


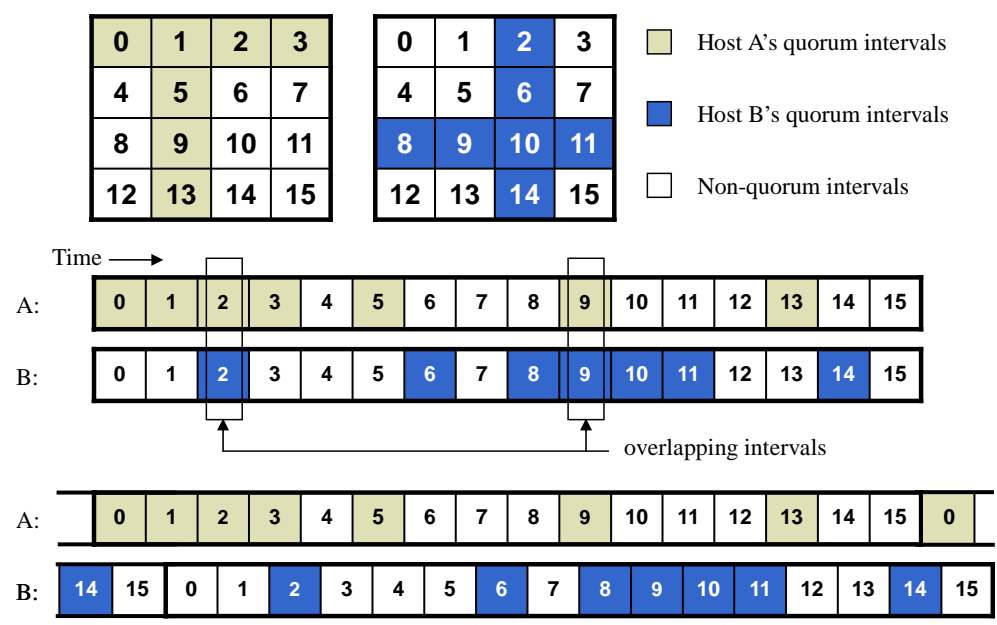

Figure 3: Arrangement of quorum intervals based on the grid quorum system in [25].

column. In the middle, we show the case where $A$ 's and $B$ 's clocks are perfectly synchronized, in which case intervals 2 and 9 of $A$ and $B$ are fully covered by each other. On the bottom, we show the case where $A$ and $B$ are asynchronous in clocks. The beacon windows of intervals 0 and 13 of $A$ are fully covered by the duration when $B$ is active. On the contrary, the beacon windows of intervals 2 and 8 of $B$ are fully covered by the duration when $A$ is active.

\subsection{Problem Statement}

The arrangement of quorum intervals in [25] is in fact based on the grid quorum system [15]. This leads to the following interesting question: Can one simply take any quorum system, which is a collection of pairwise non-disjoint sets, and apply it to solve the asynchronous power-saving problem in MANET? The answer is negative, due to the following counterexample: Let's number each host's beacon intervals by 0,1 , and 2 repeatedly, and let $\{\{0\}\}$ be the quorum system. Hence, each host will pick interval 0 as its quorum interval. It is evident that two hosts whose clocks drift by 1 or 2 beacon intervals will never be able to hear each other's beacons. Now, an even more interesting question arises: What kind of quorum systems is applicable to solve the asynchronous power-saving problem in MANETs?

The quorum-based power-saving (QPS) problem is formally defined as follows. We are given a universal set $U=\{0, \ldots, n-1\}, n \geq 2$, which represents a set of consecutive beacon intervals of mobile hosts. The goal is to determine under $U$ a quorum system $\mathcal{Q}$, which is a collection of pairwise non-disjoint subsets of $U$, each called a quorum, such that each mobile host has freedom to pick any quorum $G \in \mathcal{Q}$ to contain all its quorum intervals (the beacon intervals not in $G$ are thus non-quorum intervals). The quorum system $\mathcal{Q}$ has to guarantee that for any two arbitrarily time-asynchronous hosts $A$ and $B$, host $A$ 's beacon windows are fully covered by host $B$ 's active durations at least once in every $n$ consecutive beacon intervals, and vice versa. 


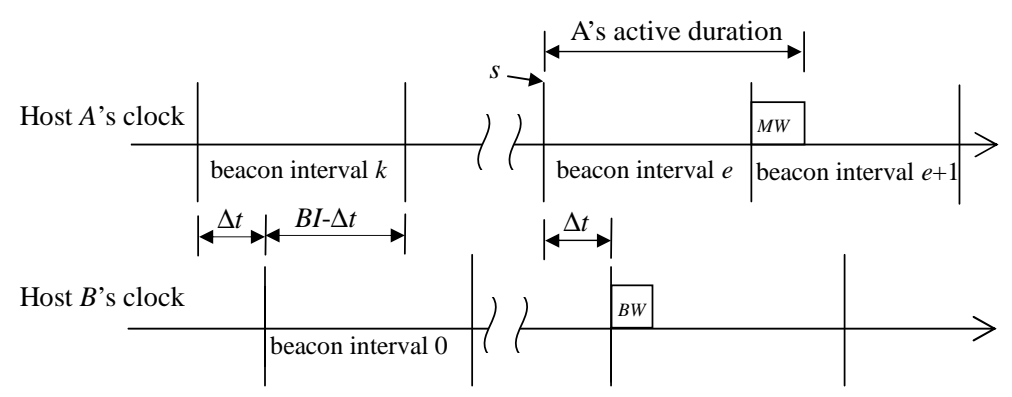

Figure 4: Timing drift of clocks of two asynchronous hosts.

\section{Quorum Systems for the QPS Problem}

Definition 1 Given a universal set $U=\{0, \ldots, n-1\}$, a quorum system $\mathcal{Q}$ under $U$ is a collection of non-empty subsets of $U$, each called a quorum, which satisfies the intersection property:

$$
\forall G, H \in \mathcal{Q}: G \cap H \neq \emptyset
$$

For example, $\mathcal{Q}=\{\{0,1\},\{0,2\},\{1,2\}\}$ is a quorum system under $U=\{0,1,2\}$.

Definition 2 Given a non-negative integer $i$ and a quorum $H$ in a quorum system $\mathcal{Q}$ under $U=\{0, \ldots, n-$ $1\}$, we define $\operatorname{rotate}(H, i)=\{j+i(\bmod n) \mid j \in H\}$.

Definition 3 A quorum system $\mathcal{Q}$ under $U=\{0, \ldots, n-1\}$ is said to have the rotation closure property if

$$
\forall G, H \in \mathcal{Q}, i \in\{0, \ldots, n-1\}: G \cap \operatorname{rotate}(H, i) \neq \emptyset
$$

For instance, the quorum system $\mathcal{Q}=\{\{0,1\},\{0,2\},\{1,2\}\}$ under $\{0,1,2\}$ has the rotation closure property. However, the quorum system $\mathcal{Q}^{\prime}=\{\{0,1\},\{0,2\},\{0,3\},\{1,2,3\}\}$ under $\{0,1,2,3\}$ has no rotation closure property because $\{0,1\} \cap \operatorname{rotate}(\{0,3\}, 3) \neq \emptyset$.

The following theorem connects quorum systems to the QPS problem.

Theorem 1 If $\mathcal{Q}$ is a quorum system satisfying the rotation closure property, $\mathcal{Q}$ is a solution to the QPS problem.

Proof. Let $A$ and $B$ be two asynchronous PS hosts in a MANET which choose $G$ and $H \in \mathcal{Q}$ to represent their quorum intervals, respectively. Without loss of generality, let $A$ 's clock lead $B$ 's clock by $k \times B I+\triangle t$, where $B I$ is the length of one beacon interval, $k<n$ is a non-negative integer, and $0 \leq \Delta t<B I$. This is illustrated in Fig. 4. First, we show that $B$ 's beacon window is fully covered by $A$ 's active durations at least once every $n$ beacon intervals. The pattern $H$ of $B$ is in fact $\operatorname{rotate}(H, k)$ from $A$ 's point of view, with an extra delay of $\triangle t$. Note that in the following discussion, time always refers to $A$ 's clock. By the rotation closure property of $\mathcal{Q}, G \cap \operatorname{rotate}(H, k) \neq \emptyset$. Let $e$ be any element in $G \cap \operatorname{rotate}(H, k)$ and let $s$ be the starting time of $A$ 's interval $e$. Also, let $B W$ and $M W$ be the lengths of one beacon window and one 
MTIM window, respectively. Taking into account the next interval $e+1$, we know that $A$ is active from $s$ to $s+B I+M W$. Since $B$ 's beacon window falls in the range $[s+\triangle t, s+\triangle t+B W]$ and $B W \leq M W$, it is easy to see that for any value of $\triangle t,[s+\triangle t, s+\Delta t+B W] \subseteq[s, s+B I+M W]$. So this part is proved.

Next, we show the reverse direction that $A$ 's beacon window is

fully covered by $B$ 's active durations at least once every $n$ beacon intervals. We first observe that if $0<\triangle t<B I$, the pattern $G$ of $A$ is $\operatorname{rotate}(G, n-k-1)$ from $B$ 's point of view, with an extra delay of $B I-\triangle t$ (note that $0<B I-\triangle t<B I)$. We also observe that if $\triangle t=0$, the pattern $G$ is rotate $(G, n-k)$ with 0 delay from $B$ 's point of view. Thus, a proof similar to that in the last paragraph can be applied to prove the reverse direction by exchanging $A$ and $B$ and substituting $\triangle t$ with $B I-\triangle t$.

It is important to note that the number of quorum intervals reflects the power consumption of PS hosts since quorum intervals are more energy-consuming (recall that a PS host needs to send a beacon and remains active in each quorum interval). Given a fixed $n$, the cost can be measured by the sizes of quorums in the quorum system. It is desirable that the quorum sizes are as small as possible. In the following theorem, we derive a lower bound on quorum sizes for any quorum system satisfying the rotation closure property. A quorum system is said to be optimal if the sizes of all its quorums meet the lower bound.

Theorem 2 Let $\mathcal{Q}$ be a quorum system under $\{0, \ldots, n-1\}$. If $\mathcal{Q}$ satisfies the rotation closure property, then any quorum in $\mathcal{Q}$ must have a cardinality $\geq \sqrt{n}$.

Proof. Let $H=\left\{h_{1}, \ldots, h_{k}\right\}$ be any quorum in $\mathcal{Q}$, where $0<k<n$. There are two cases.

Case 1) $H \neq \operatorname{rotate}(H, i)$ for any $i \neq n(\bmod n)$ : Since $h_{1}, h_{2}, \ldots, h_{k}$ are distinct elements, it is clear that $h_{1}+i, h_{2}+i, \ldots, h_{k}+i(\bmod n)$ are also distinct for any $i=1 . . n-1$. So, $|\operatorname{rotate}(H, i)|=k$. Let's call $\operatorname{rotate}(H, i), i=1 . . n-1$, the rotating quorums of $H$. For each element $h_{j} \in H$, it belongs to exactly $k-1$ rotating quorums of $H$, namely $\operatorname{rotate}\left(H,\left(h_{j}-h_{j^{\prime}}\right) \bmod n\right)$ for every $h_{j^{\prime}} \neq h_{j}$. By the rotation closure property, $H$ must contain at least one element from each of the $n-1$ rotating quorums of $H$. Since each element appears in exactly $k-1$ rotating quorums of $H$ and there are $k$ elements in $H$, we have $k(k-1) \geq n-1$, which implies $k>\sqrt{n}$. Thus, the theorem holds for case 1.

Case 2) $H=\operatorname{rotate}(H, i)$ for some $i \neq n(\bmod n)$ : Let $d$ be the smallest integer such that $H=$ $\operatorname{rotate}(H, d)$. It is a simple result in number theory that $n$ is a multiple of $d$. So it can be concluded that $H=\operatorname{rotate}(H, d)=\operatorname{rotate}(H, 2 d)=\operatorname{rotate}(H, 3 d)=\cdots=\operatorname{rotate}(H, n-d)$. That is, when mapping the quorum elements of $H$ onto the time axis, $H$ can be regarded as $n / d$ equivalent segments, each of length $d$. In fact, from $H$, we can define a smaller quorum

$$
H^{\prime}=\{j \bmod d \mid j \in H\}
$$

under the universal set $\{0, \ldots, d-1\}$. Intuitively, on the time axis, $H$ can be considered as a concatenation of $n / d$ copies of $H^{\prime}$. Since $H \cap \operatorname{rotate}(H, i) \neq \emptyset$, we can conclude that $H^{\prime} \cap \operatorname{rotate}\left(H^{\prime}, i\right) \neq \emptyset$ for any $i$ under 
modulo- $d$ arithmetic. So $\left\{H^{\prime}\right\}$ is also a quorum system satisfying the rotation closure property under the universal set $\{0, \ldots, d-1\}$. We can apply the result in case 1 and infer that $\left|H^{\prime}\right| \geq \sqrt{d}$. It follows that $|H|=(n / d)\left|H^{\prime}\right| \geq(n / d) \sqrt{d}>\sqrt{n}$.

\section{Quorum Systems with the Rotation Closure Property}

Although there are volumes of works devoted to quorum systems, none of them discusses the rotation closure property to the best of our knowledge. In this section, we prove that the grid quorum system [15], the torus quorum system [12], the cyclic quorum system [14], and the finite projective plane quorum system [15] are all optimal or near optimal quorum systems (in terms of quorum sizes) satisfying the rotation closure property.

\subsection{The Grid Quorum System}

The grid quorum system [15] arranges elements of the universal set $U=\{0, \ldots, n-1\}$ as a $\sqrt{n} \times \sqrt{n}$ array. A quorum can be any set containing a full column plus a full row of elements in the array. Thus, each quorum has a near optimal size of $2 \sqrt{n}-1$. As noted above, the work in [25] adopts the grid quorum system. Below, we prove the rotation closure property for the grid quorum system. The theorem, when accompanied with Theorem 1, can simplify the lengthy correctness proof of the work in [25], which needs to deal with complicated timing relation between quorum and non-quorum intervals among different asynchronous hosts.

Theorem 3 The grid quourm system satisfies the rotation closure property.

Proof. Let $\mathcal{Q}$ be a grid quorum system. Let $H \in \mathcal{Q}$, which contains all elements on the column $c$ of the array, namely $c, c+\sqrt{n}, \ldots, c+(\sqrt{n}-1) \sqrt{n}$, where $0 \leq c<n$ (note that we number columns from 0 to $\sqrt{n}-1)$. Now observe that rotate $(H, i)$ must contain all elements on

column $(c+i)(\bmod \sqrt{n})$. It follows that rotate $(H, i)$ must have intersection with any quorum $G \in \mathcal{Q}$ because $G$ must contain a full row in the array.

\subsection{The Torus Quorum System}

Similar to the grid quorum system, the torus quorum system [12] also adopts an array structure. The universal set is arranged as a $t \times w$ array, where $t w=n$. Following the concept of torus, the rightmost column (resp., the bottom row) in the array are regarded as wrapping around back to the leftmost column (resp., the top row). A quorum is formed by picking any column $c, 0 \leq c \leq w-1$, plus $\lfloor w / 2\rfloor$ elements, each of which falls in any position of column $c+i, i=1 . .\lfloor w / 2\rfloor$. Fig. 5 illustrates the construction of two torus quorums $G$ and $H$ under $U=\{0, \ldots, 17\}$ with $t=3$ and $w=6$. $G$ is formed by picking the second column plus three elements, each from one of the third, fourth, and fifth columns. $H$ is formed by picking 


\begin{tabular}{|c|c|c|c|c|c|c|}
\hline 0 & 1 & 2 & 3 & 4 & 5 & $\square$ Quorum $G$ \\
\hline 6 & 7 & 8 & 9 & 10 & 至1 & 目 Quorum $H$ \\
\hline 12 & 13 & 74 & 15 & 16 & 17 & $\begin{array}{l}\text { 目 Intersection } \\
\text { of } G \text { and } H\end{array}$ \\
\hline
\end{tabular}

Figure 5: Two quorums of the torus quorum system in a $3 \times 6$ torus.

the sixth column plus three elements, each from one of the first, second, and third columns. $G$ and $H$ intersect at element 7 .

As shown in [12], if we let $t=w / 2$, the quorum size will be $\approx \sqrt{2 t w}=\sqrt{2 n}$, which is near optimal. By equating $n$, the torus quorum size is about $1 / \sqrt{2}$ that of the grid quorum size. Below, we prove the rotation closure property for the torus quorum system.

Theorem 4 The torus quorum system satisfies the rotation closure property.

Proof. Let $\mathcal{Q}$ be a torus quorum system formed by a $t \times w$ array and $H \in \mathcal{Q}$ be a quorum containing column $c$. By definition, $H$ also contains another $\lfloor w / 2\rfloor$ elements, each from one of the $\lfloor w / 2\rfloor$ succeeding columns of column $c$. Clearly, rotate $(H, i)$ still has the torus quorum structure for an arbitrary $i$. It follows that for any $G \in \mathcal{Q}, G \cap \operatorname{rotate}(H, i) \neq \emptyset$.

\subsection{The Cyclic Quorum System}

The cyclic quorum systems [14] are constructed from the difference sets as defined below.

Definition 4 A subset $D=\left\{d_{1}, d_{2}, \ldots, d_{k}\right\}$ of $Z_{n}$ is called a difference set under $Z_{n}$ if for every $e \neq 0(\bmod$ $n)$ there exists at least two elements $d_{i}$ and $d_{j} \in D$ such that $d_{i}-d_{j}=e(\bmod n)$.

Definition 5 Given any difference set $D=\left\{d_{1}, d_{2}, \ldots, d_{k}\right\}$ under $Z_{n}$, the cyclic quorum system defined by $D$ is $\mathcal{Q}=\left\{G_{1}, G_{2}, \ldots, G_{n}\right\}$, where $G_{i}=\left\{d_{1}+i, d_{2}+i, \ldots, d_{k}+i\right\}(\bmod n), i=0, \ldots, n-1$.

For example, $D=\{0,1,2,4\} \subseteq Z_{8}$ is a difference set under $Z_{8}$ since each $e=1 . .7$ can be generated by taking the difference of two elements in $D$. Given $D, \mathcal{Q}=\left\{G_{0}=\{0,1,2,4\}, G_{1}=\{1,2,3,5\}, G_{2}=\right.$ $\left.\{2,3,4,6\}, G_{3}=\{3,4,5,7\}, G_{4}=\{4,5,6,0\}, G_{5}=\{5,6,7,1\}, G_{6}=\{6,7,0,2\}, G_{7}=\{7,0,1,3\}\right\}$ is a cyclic quorum system under $Z_{8}$.

Given any $n$, a difference set as small as $k$ can be found when $k(k-1)+1=n$ and $k-1$ is a prime power. Such a difference set is called the Singer difference set [4]. For example, the sets $\{1,2,4\}$ under $Z_{7}$ and $\{1,2,4,9,13,19\}$ under $Z_{31}$ are Singer difference sets. Note that in this case the quorum size $k$ meets the lower bound in Theorem 2. So cyclic quorum systems defined by the Singer difference sets are optimal. Reference [14] had conducted exhausted searches to find the minimal difference sets under $Z_{n}$ for $n=4 . .111$. The results are useful here to construct near-optimal cyclic quorum systems. 
Theorem 5 The cyclic quorum system satisfies the rotation closure property.

Proof. Let $H$ be a quorum in the cyclic quorum system $\mathcal{Q}$ generated from the difference set $D=$ $\left\{d_{1}, d_{2}, \ldots, d_{k}\right\}$. By definition, rotate $(H, i)$ is also a quorum in $\mathcal{Q}$ for any $i$. Then by the intersection property, the theorem holds.

\subsection{The Finite Projective Plane Quorum System}

The finite projective plane (FPP) quorum system [15] arranges elements of the universal set $U=\{0, \ldots, n-$ $1\}$ as vertices on a hypergraph called the finite projective plane, which has $n$ vertices and $n$ edges, such that each edge is connected to $k$ vertices and two edges have exactly one common vertex. (Note that the hypergraph is a generalization of typical graphs, where each edge is connected to only two vertices.) A quorum can be formed by the set of all vertices connected by the edge, and thus has a size of $k$. It has been shown in [15] that a FPP can be constructed when $n=k(k-1)+1$ and $k-1$ is a prime power. Otherwise, the FPP may or may not exist. In [14], the FPP construction is associated to the construction of Singer difference sets, and it is shown that the FPP quorum system can be regarded as a special case of the cyclic quorum system when $n=k(k-1)+1$ and $k-1$ is a prime power. It follows that FPP quorum systems also own the rotation closure property, and are optimal, when existing.

\subsection{Quorum Systems with One Quorum}

In this subsection, we discuss the rotation closure property for those quorum systems with only one quorum. The result has strong connection to the difference sets, and can help identify the quorum systems that are solution to the QPS problem.

Theorem 6 Let $\mathcal{Q}=\{H\}$ be a quorum system under $U=\{0, \ldots, n-1\}$. $\mathcal{Q}$ satisfies the rotation closure property if and only if $H$ is a difference set of $Z_{n}$.

Proof. For the "if" part, let $H$ be a difference set of $Z_{n}$. For any $i$, there must exist two elements $h_{x}, h_{y} \in H$ such that $h_{x}-h_{y}=i$. It follows that $h_{x}=h_{y}+i \in \operatorname{rotate}(H, i) \cap H$. So rotate $(H, i) \cap H \neq \emptyset$ for any $i$.

For the "only if" part, suppose for contradiction that $H$ is not a difference set of $Z_{n}$. Then there exists an $i \neq 0$ such that $h_{x}-h_{y} \neq i$ for all possible combinations of $h_{x}$ and $h_{y}$ in $H$. Since rotate $(H, i)=$ $\left\{\left(h_{y}+i\right) \bmod n \mid h_{y} \in H\right\}$, it follows that $H \cap \operatorname{rotate}(H, i)=\emptyset$, a contradiction.

Corollary 1 Let $\mathcal{Q}$ be a quorum system under $U=\{0, \ldots, n-1\}$. $\mathcal{Q}$ does not satisfies the rotation closure property if at least one quorum in $\mathcal{Q}$ is not a difference set under $Z_{n}$.

Theorem 6 says that if a quorum system has a difference set being its sole quorum, it satisfies the rotation closure property and is thus a solution to the QPS problem. Such a quorum system has the 
practical advantage that it is very easy to maintain since it has only one quorum to keep. For example, from each of the minimal difference sets found in [14] (for $n=4 . .111$ ), a solution to the QPS problem exists by simply putting the different set as the single quorum in the quorum system. On the contrary, when $n$ is too large such that exhausted searches (as in [14]) are prohibited, we can pick any quorum $G$ in the quorum systems with the rotation closure property. Then $G$ is a difference set by the contraposition of Corollary 1. For example, from the torus quorum system, we can quickly find a lot of near-optimal difference sets by arranging numbers from 0 to $n-1$ as an array. Note that in situations when $n$ can not be divided into a product of $t$ and $w$, we can always add a "virtual element" on the array, as proposed in [15], to solve the problem. For example, when $n=13$, we can make a $2 \times 7$ array with the last position filled by 0 as the virtual element.

\section{$5 \quad$ An Adaptive QPS Protocol}

All the quorum systems discussed above ensure that given a fixed $n$, two asynchronous mobile hosts picking any two quorums have at least one intersection in their quorums. It would be desirable to have an adaptive solution in the sense that the number of intersecting elements can be dynamically adjusted. One of the main reasons to do so would be to adjust this value to adapt to host mobility. Intuitively, the number of beacons that two hosts can hear from each other is proportional to the number of intersecting elements. Thus, a host with higher mobility may like to have more intersections with its neighboring hosts so as to be more environment-sensitive. On the contrary, a host with lower mobility may not need to intersect in so many elements with its neighbors so as to save more energy. The proposed solution is adaptive in this sense.

We assume that a host is able to calculate its mobility levels, either through attaching a GPS device, or simply by evaluating the number of hosts that are detected to leave/enter the host's radio coverage. We leave this as an independent issue, and only focus on the design of adaptive quorum systems to meet our goal.

The proposed solution is basically an extension of the torus quorum system, and is thus called the extended torus (e-torus) quorum system. An e-torus quorum system is also defined based on two given integers $t$ and $w$ such that $U=\{0,1, \ldots, t w-1\}$ is the universal set. Elements of $U$ are arranged in a $t \times w$ array. Below, we use $[x, y]$ as an array index, $0 \leq x<t$ and $0 \leq y<w$.

Definition 6 On a $t \times w$ array, a positive half diagonal starting from position $[x, y]$, where $0 \leq x<t$ and $0 \leq y<w$, consists of element $[x, y]$ plus $\lfloor w / 2\rfloor$ elements $[(x+i) \bmod t,(y+i) \bmod w]$, for $i=1 . .\lfloor w / 2\rfloor$.

A negative half diagonal starting from position $[x, y]$ consists of element $[x, y]$ plus $\lceil w / 2\rceil-1$ elements $[(x+i) \bmod t,(y-i) \bmod w]$, for $i=1 . .\lceil w / 2\rceil-1$.

Intuitively, a positive (resp., negative) half diagonal is a partial diagonal on the array starting from 


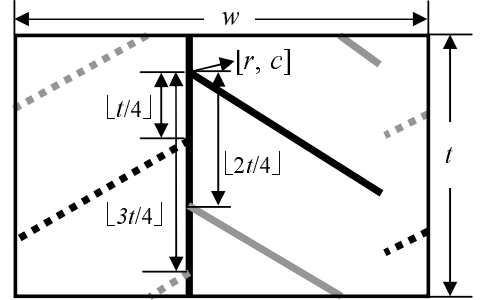

(a)

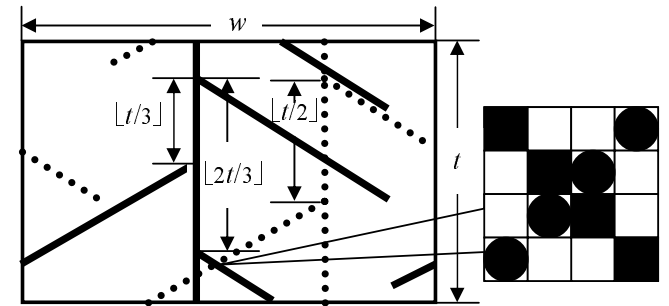

(b)

Figure 6: (a) the "Christmas tree" structure of an e-torus(4) quorum, and (b) the intersection of an e-torus(2) quorum and an e-torus(3) quorum.

the array index $[x, y]$ with a length $\lfloor w / 2\rfloor+1$ (resp., $\lceil w / 2\rceil$ ). A positive diagonal goes in the southeast direction, while a negative one goes in the southwest direction. The diagonal is slightly different from typical "diagonal" in matrix algebra in that the array is not necessarily square and that the torus has the wrap-around property.

Definition 7 Given any integer $k \leq t$, a quorum of an $e$-torus $(k)$ quorum system is formed by picking any position $[r, c]$, where $0 \leq r<t$ and $0 \leq c<w$, such that the quorum contains all elements on column $c$ plus $k$ half diagonals. These $k$ half diagonals alternate between positive and negative ones, and start from the following positions:

$$
\left[r+\left\lfloor i \times \frac{t}{k}\right\rfloor, c\right], \quad i=0 . . k-1 .
$$

Intuitively, each quorum in the e-torus $(k)$ quorum system looks like a Christmas tree with a trunk in the middle and $k$ branches, each as a half diagonal, alternating between positive and negative ones. Fig. 6(a) illustrates the conceptual structure of an e-torus(4) quorum.

Theorem 7 The e-torus quorum system satisfies the rotation closure property.

Proof. Since any e-torus quorum is a super set of a torus quorum, the theorem holds.

Theorem 8 Let $G$ be an e-torus $\left(k_{1}\right)$ quorum and $H$ be an e-torus $\left(k_{2}\right)$ quorum derived from the same array. For any integers $i$ and $j,|\operatorname{rotate}(G, i) \cap \operatorname{rotate}(H, j)| \geq\left\lfloor\left(k_{1}+k_{2}\right) / 2\right\rfloor$.

Proof. This theorem can be easily observed from the geometric structure of the e-torus quorum system (by evaluating the number of branches intersecting with the trunks of the Christmas trees).

For example, Fig. 6(b) shows how an e-torus(3) quorum and an e-torus(2) quorum intersect with each other. The intersecting elements are guaranteed to appear in the trunks of the "Christmas trees." Note that two branches from two e-torus quorums may "cross with" each other, but intersection is not necessarily guaranteed (from the geometric structures of branches, it does look like that they are guaranteed to intersect). The reason is illustrated in the zoomed-in part in Fig. 6(b), where the two branches just 


\begin{tabular}{|c|c|c|c|c|c|c|c|c|c|c|c|c|c|}
\hline \multicolumn{11}{|c|}{]$: e-t o r u s(2)$ 目:e-torus(1) } & \multicolumn{3}{|c|}{$\mathrm{NS}=B I \times n$} \\
\hline 0 & 1 & 2 & 3 & 4 & 5 & 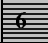 & 7 & 8 & 9 & 10 & $\mathbf{1 1}$ & 12 & 13 \\
\hline 14 & 15 & 16 & & 18 & 1 & 卢 & 21 & 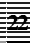 & 23 & 24 & 25 & 26 & 27 \\
\hline 28 & 29 & 30 & 31 & 32 & 33 & 臨 & 35 & 36 & 厚 & 38 & \begin{tabular}{|l|}
39 \\
\end{tabular} & 40 & 41 \\
\hline 42 & 43 & 44 & 45 & 46 & 47 & 18 & 49 & $\mathbf{5 0}$ & 51 & $\sqrt{58}$ & 53 & 54 & 55 \\
\hline 56 & 57 & 58 & 59 & 60 & 61 & 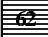 & 63 & 64 & 65 & 66 & 6 & 68 & 69 \\
\hline 70 & 71 & 72 & 73 & 74 & 75 & 常 & 77 & 78 & 79 & 80 & $\mathbf{8 1}$ & 5 & 83 \\
\hline 84 & 85 & 86 & 87 & 88 & 89 & 可位 & 91 & 92 & 93 & 94 & 95 & 96 & 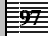 \\
\hline
\end{tabular}

\begin{tabular}{|c|c|c|c|c|c|c|c|c|c|c|c|c|c|}
\hline \multicolumn{10}{|c|}{ :e-torus(3) 四:e-torus(1) } & \multicolumn{4}{|c|}{$\mathrm{NS}=B I \times n-1$} \\
\hline 0 & 1 & 2 & 3 & 官霓 & 5 & 6 & 7 & 8 & 9 & 衰 & 11 & 12 & 13 \\
\hline 14 & 15 & 16 & 17 & 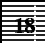 & 19 & 20 & 21 & 22 & 23 & 24 & 5 & 26 & 27 \\
\hline 28 & 29 & 30 & $\boldsymbol{J}$ & 哺 & 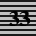 & 34 & 35 & 36 & 37 & 38 & 39 & 40 & 41 \\
\hline 42 & 43 & 44 & 45 & 更 & 47 & 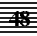 & 49 & 50 & 51 & 52 & 53 & 54 & 55 \\
\hline 56 & 57 & 58 & 59 & 6 & 61 & 62 & 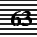 & 64 & 65 & 66 & 67 & 68 & 69 \\
\hline 70 & 71 & 72 & 73 & 官唔 & 75 & 76 & 77 & 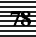 & 79 & 80 & 81 & 82 & 83 \\
\hline 84 & 85 & 86 & 87 & 88 & 89 & 90 & 91 & 92 & 59 & 94 & 95 & 96 & 97 \\
\hline
\end{tabular}

\begin{tabular}{|c|c|c|c|c|c|c|c|c|c|c|c|c|c|}
\hline \multicolumn{8}{|c|}{ ]:e-torus(3) 目:e-torus(1) } & \multicolumn{6}{|c|}{$\mathrm{NS}=B I \times\left(n-\left\lfloor 2 t / k_{1}\right\rfloor \times w\right)$} \\
\hline 0 & 1 & 2 & 2 & 4 & 5 & 西 & 高 & 8 & 9 & 10 & $\mathbf{1 1}$ & 12 & 13 \\
\hline 14 & 15 & & & 18 & 19 & 泟 & 21 & 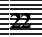 & 23 & 24 & 25 & 26 & 27 \\
\hline 28 & 29 & & & 32 & 33 & 资 & 35 & 36 & 5 & 38 & 39 & 40 & 41 \\
\hline 42 & 43 & 44 & 45 & 46 & 71 & 年 & 49 & 50 & 51 & 5 & 53 & 54 & 55 \\
\hline 56 & 57 & 58 & 59 & 60 & 61 & 6 & 63 & 64 & 65 & 66 & 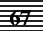 & 68 & 69 \\
\hline 70 & 71 & 72 & 73 & 74 & 75 & $\sqrt{176}$ & 77 & 78 & 79 & 80 & 81 & 82 & 83 \\
\hline 84 & 85 & 86 & 87 & 88 & 89 & (e) & 91 & 92 & 93 & 94 & 95 & 96 & 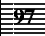 \\
\hline
\end{tabular}

\begin{tabular}{|c|c|c|c|c|c|c|c|c|c|c|c|c|c|}
\hline \multicolumn{9}{|c|}{ :e-torus(2) 目:e-torus(2) } & \multicolumn{5}{|c|}{$\mathrm{NS}=B I \times(n-w+1)$} \\
\hline 0 & 1 & 2 & 3 & 4 & 5 & 㢄高 & 7 & 8 & 㹂 & 10 & 11 & 12 & 13 \\
\hline 14 & 1 & & & 18 & 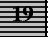 & 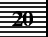 & 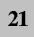 & 22 & 23 & 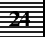 & 25 & 26 & 27 \\
\hline 28 & 29 & 30 & 31 & 3 & 33 & 84 & 35 & 36 & 37 & 38 & 翟 & 40 & 41 \\
\hline 42 & 43 & 44 & 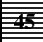 & 46 & 47 & 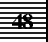 & 49 & 50 & 51 & 52 & 53 & 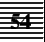 & 55 \\
\hline 56 & 57 & 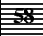 & 59 & 60 & 61 & $\bar{E}$ & 63 & 64 & 65 & 66 & 67 & 68 & 69 \\
\hline 70 & $\overline{17}$ & 72 & 73 & 74 & 75 & 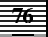 & 7 & 78 & 79 & 80 & 81 & 82 & 83 \\
\hline 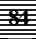 & 85 & 86 & 87 & 88 & 89 & 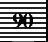 & & 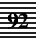 & 93 & 94 & 95 & 96 & 97 \\
\hline
\end{tabular}

Figure 7: Analysis of neighbor sensibility of an e-torus $\left(k_{1}\right)$ and an e-torus $\left(k_{2}\right)$ quorum systems under a $7 \times 14$ torus.

miss each other on the array. Also note that by our arrangement, the intersecting elements of two e-torus quorums are unlikely to concentrated in certain areas of the array. Instead, they will be spread evenly over the trunks. This is a desirable property because it implies that the quorum intervals that two mobile hosts may detect each other will be spread evenly over the time axis.

Based on the above features, we propose an adaptive QPS protocol as follows. We can rank a host's mobility into $k$-levels, where level 1 means the lowest mobility, and level $k$ means the highest mobility. Whenever a host determines that its mobility falls within level $i(1 \leq i \leq k)$, it adjusts its quorum intervals based on any e-torus $(i)$ quorum. Consequently, a host can dynamically adjust its sensibility to the environment change in its neighborhood.

\section{Performance Comparison and Simulation Results}

\subsection{Analytical Comparison}

In this subsection, we compare the proposed quorum-based protocols analytically. We evaluate the active ratio, which is defined to be the number of quorum intervals over $n$ (the size of universal set), and the neighbor sensibility (NS), which is the worst-case delay for a PS host to detect the existence of a newly approaching PS host in its neighborhood. The NS of the grid quorum system is $B I \times(n-\sqrt{n}+1)$, which happens when two quorums intersect at indices $(i, j)$ and $(i+1, j-1)$ of the array. The NS of two e-torus quorum systems e-torus $\left(k_{1}\right)$ and e-torus $\left(k_{2}\right)$ under a $t \times w$ torus is discussed below (refer to Fig. 7 for illustration). When $\left(k_{1}=k_{2}=1\right),\left(k_{1}=2 \wedge k_{2}=1\right)$, or $\left(k_{1}=1 \wedge k_{2}=2\right)$, the two quorums may intersect at only one interval, so NS is $B I \times n$. For $\left(k_{1}=3 \wedge k_{2}=1\right)$ or $\left(k_{1}=1 \wedge k_{2}=3\right)$, the NS is $B I \times(n-1)$, 
Table 2: Active ratios and neighbor sensitivity of quorum-based protocols.

\begin{tabular}{|c|c|c|}
\hline Quorum system & Active ratio & Neighbor sensibility \\
\hline Grid & $\approx \frac{2}{\sqrt{n}}$ & $B I \times(n-\sqrt{n}+1)$ \\
\hline Torus & $\approx \frac{\sqrt{2}}{\sqrt{n}}$ & $B I \times n$ \\
\hline Cyclic & $\approx \frac{1}{\sqrt{n}}$ & $B I \times n$ \\
\hline Finite projective plane & $\begin{array}{l}\frac{k}{n}, \text { where } k(k-1)+1=n, \\
\text { and } k-1 \text { is a prime power }\end{array}$ & $B I \times n$ \\
\hline $\begin{array}{l}\text { e-torus }(k), \\
\text { (under } t \times w \text { torus) }\end{array}$ & $\begin{array}{l}\frac{s}{n}, \text { where } s=t+w\left\lfloor\frac{k}{2}\right\rfloor+ \\
r\left\lfloor\frac{w-1}{2}\right\rfloor, r=0 \text { if } k \text { is even and } \\
r=1 \text { if } k \text { is odd }\end{array}$ & $\begin{array}{l}\text { For e-torus }\left(k_{1}\right) \text { and e-torus }\left(k_{2}\right): \\
\text { - } B I \times n, \text { for }\left(k_{1}=k_{2}=1\right),\left(k_{1}=\right. \\
\left.2 \wedge k_{2}=1\right) \text {, or }\left(k_{1}=1 \wedge k_{2}=2\right) \\
\text { - } B I \times(n-1), \text { for }\left(k_{1}=3 \wedge k_{2}=1\right) \text { or } \\
\left(k_{1}=1 \wedge k_{2}=3\right) \\
\text { - } B I \times\left(n-\left\lfloor 2 t / k_{1}\right\rfloor\right), \text { for }\left(k_{1}=1 \wedge k_{2}=\right. \\
4) \text { or }\left(k_{1}=4 \wedge k_{2}=1\right) \\
\text { - less than } B I \times\left(n-\left\lfloor 2 t / k_{1}\right\rfloor\right), \text { for }\left(k_{1}>\right. \\
\left.4 \wedge k_{2}=1\right) \text { or }\left(k_{1}=1 \wedge k_{2}>4\right) \\
\text { - } B I \times(n-w+1), \text { for }\left(k_{1}=2 \wedge k_{2}=2\right) \\
\text { - less than } B I \times(n-w+1), \text { for }\left(k_{1} \geq\right. \\
\left.2 \wedge k_{2}>2\right) \text { or }\left(k_{1}>2 \wedge k_{2} \geq 2\right)\end{array}$ \\
\hline
\end{tabular}

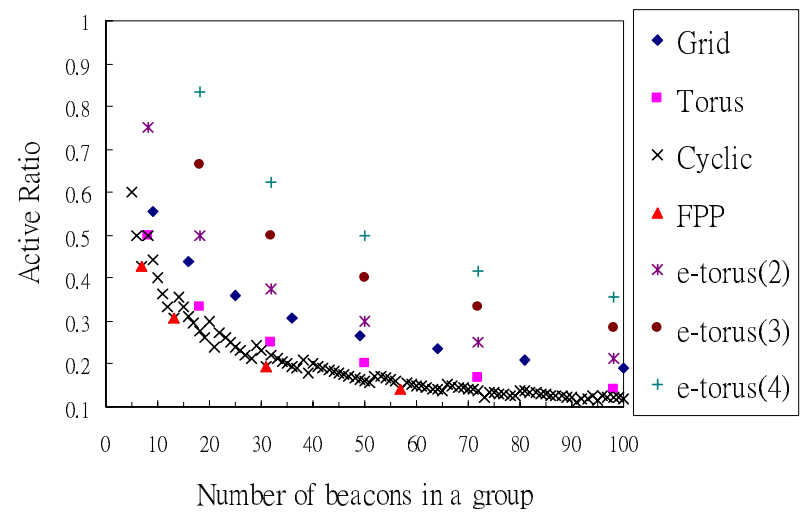

Figure 8: Active ratios of different quorum systems for $n=5 . .100$.

which happens when two quorums intersect at two consecutive quorum intervals. For $\left(k_{1}=1 \wedge k_{2}=4\right)$ or $\left(k_{1}=4 \wedge k_{2}=1\right)$, the NS is $B I \times\left(n-\left\lfloor 2 t / k_{1}\right\rfloor\right)$, which happens when the intersections fall in one column. Table 2 contains the NS of other cases. Table 2 also summarizes the active ratio and neighbor sensibility of the proposed quorum-based protocols. Fig. 8 further demonstrates the active ratio of the proposed protocols for $n=5 . .100$. The cyclic quorum performs the best in terms of active ratio. The FPP quorum system, when available, represents the optimal solution.

\subsection{Simulation Results}

In this subsection, we compare the proposed power-saving protocols through a simulator written in C. An area of size $1000 \mathrm{~m} \times 1000 \mathrm{~m}$ is simulated. Each host has an antenna with a transmission rate of $2 \mathrm{M}$ bits/sec and a transmission radius of 250 meters, and has an initial battery energy of 100 Joules. The MAC part 
basically follows the IEEE 802.11 standard [11], except the power management part. Routes with random sources/destinations are generated, and the AODV routing protocol [16] is adopted. Four parameters are tunable in our simulations:

- Mobility: Host mobility follows the random way-point model, with pause time of 20 seconds. When moving, a host's speed can range in $0 \sim 20 \mathrm{~m} / \mathrm{sec}$.

- Traffic load: Routes are generated by a Poisson distribution with rates between $1 \sim 4$ routes/sec. For each route, 10 packets, each of size $1 \mathrm{~K}$ bytes, are sent.

- Beacon interval: The length of one beacon interval is $100 \sim 400 \mathrm{~ms}$.

- Number of hosts: The total number of mobile hosts in the MANET is $50 \sim 200$ hosts.

Three performance metrics are measured in the simulations:

- Survival ratio: the number of surviving hosts (with non-zero energy) over the total number of hosts.

- Neighbor discovery time: average time to discover a newly approaching neighbor.

- Throughput: the average number of MAC-layer data packets successfully received in the network per second.

Except the survival ratio, the above metrics are evaluated up to the time when $10 \%$ of the hosts run out of energy. A host can go to the PS mode when it does not serve as a source, destination, or relay of any route. A broadcast (such as the AODV route request message) may need to be sent multiple times if the sending host finds that some of its neighbors are in the PS mode [25]. This is necessary because these PS hosts may wake up at different times and we need multiple transmissions to cover all of them. However, once a route is established (via the notification of a route reply message), all hosts in the route have to tune to the active mode.

Table 3 summarizes the power consumption parameters used in our simulations, which are obtained from real experiments using Lucent WaveLAN cards [5]. Sending/receiving a unicast/broadcast packet of $L$ bytes has a cost $P_{\text {base }}+P_{\text {byte }} \times L$, where $P_{\text {base }}$ is the power consumption independent of packet length and $P_{\text {byte }}$ is the power consumption per byte. Unicast consumes more power than broadcast because it incurs extra control frames $(R T S, C T S$, and $A C K)$. Idle/doze represents the condition when a host has no send and receive activity and is in the active/PS mode, respectively. The traffic-related parameters are summarized in Table 4.

Below, we show how mobility, beacon interval length, traffic load, and host density affect the performance of the proposed PS protocols. We mainly compare the cyclic quorum system (which has the lowest active ratio) and the e-torus quorum system (which is more adaptive). Below, $C(n)$ stands for the cyclic 
Table 3: Power consumption parameters used in the simulation.

\begin{tabular}{|c|c|}
\hline Unicast send & $454+1.9 \times L \mu \mathrm{L} /$ packet \\
Broadcast send & $266+1.9 \times \mathrm{L} \mu \mathrm{J} /$ packet \\
Unicast receive & $356+0.5 \times L \mu \mathrm{J} /$ packet \\
Broadcast receive & $56+0.5 \times \mathrm{L} \mu \mathrm{J} /$ packet \\
Idle & $843 \mu \mathrm{J} / \mathrm{ms}$ \\
Doze & $27 \mu \mathrm{J} / \mathrm{ms}$ \\
\hline
\end{tabular}

Table 4: Traffic-related parameters used in the simulation.

\begin{tabular}{|c|c|}
\hline Unicast packet size & 1024 bytes \\
Broadcast packet size & 32 bytes \\
Beacon window size & $4 \mathrm{~ms}$ \\
MTIM window size & $16 \mathrm{~ms}$ \\
\hline
\end{tabular}

quorum system under $\{0,1, . ., n-1\}$, and $E(t \times w)$ the e-torus quorum system under a $t \times w$ torus. For the e-torus quorum system, four speed levels $(1 \sim 4)$ are assumed. A host is said to be at speed level $i$ if its speed is larger than $5(i-1) \mathrm{m} / \mathrm{sec}$ and less than or equal to $5 i \mathrm{~m} / \mathrm{sec}$. To make comparison, we also simulate an "always-active (AA)" scheme in which all hosts are active all the time.

\subsubsection{Impact of Mobility}

Mobility has a negative impact on survival ratio. Fig. 9 compares the cases when all hosts are stationary and when all hosts' moving speed $=20 \mathrm{~m} / \mathrm{sec}$. Mobility will incur higher energy consumption because hosts may spend more energy in retransmitting packets. On the contrary, mobility has very little impact on AA. However, because hosts can tune to the PS mode, $\mathrm{C}(98)$ and $\mathrm{E}(7 \times 14)$ still outperform AA significantly in terms of survival ratio. The survival ratio of $\mathrm{C}(98)$ is better than that of $\mathrm{E}(7 \times 14)$ because its active ratio is smaller.

Fig. 10 shows the impact of mobility on the neighbor discovery time. Mobility has a negative impact on neighbor discovery time for $\mathrm{C}(98)$. On the contrary, $\mathrm{E}(7 \times 14)$ can better adapt itself to mobility. We even see shorter neighbor discovery time when host mobility becomes higher (at the cost of more beacon intervals).

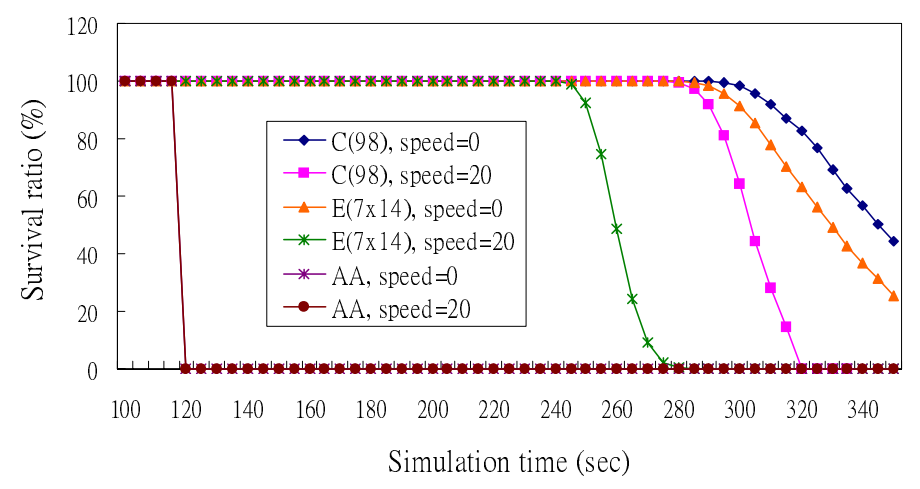

Figure 9: Host survival ratio vs. mobility (beacon interval $=100 \mathrm{~ms}, 100$ hosts, traffic load $=1$ route/sec). 


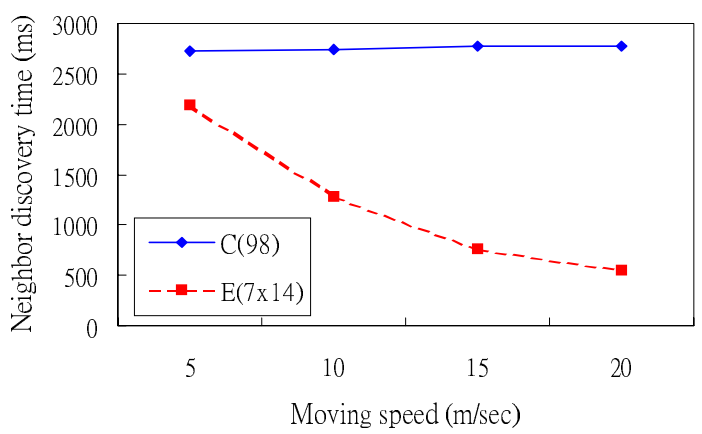

Figure 10: Neighbor discovery time vs. mobility (beacon interval $=100 \mathrm{~ms}, 100$ hosts, traffic load $=1$ route/sec).

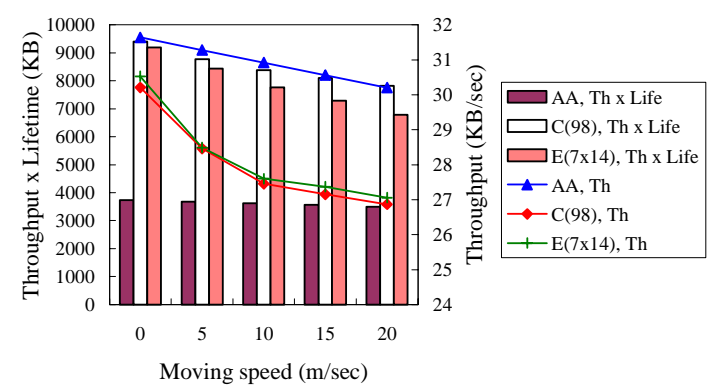

Figure 11: Throughput vs. mobility (beacon interval $=100 \mathrm{~ms}, 100$ hosts, traffic load $=1$ route/sec).

Fig. 11 shows the impact of mobility on throughput. Mobility has a negative impact on throughput for all schemes because more retransmissions are incurred as hosts move faster. The results show that $\mathrm{C}(98)$ and $\mathrm{E}(7 \times 14)$ will slightly degrade throughputs compared to AA when we allow hosts to tune to the PS mode, which is reasonable. However, the benefit is that the network can be used for much longer time, as reflected by the axis "throughput $\times$ lieftime", where the lifetime of a network is counted up to the point when $10 \%$ of hosts runs out of energy.

\subsubsection{Impact of Beacon Interval Length}

We observe the impact of beacon interval (BI) length on host survival ratio by varying the beacon interval length between 100 400 ms. Fig. 12 shows that a longer BI will slightly shorten the lifetime of the network for $\mathrm{C}(98)$ and $\mathrm{E}(7 \times 14)$. We believe that this is due to a higher transmission cost for broadcasting route request packets. However, a longer BI makes hosts conserve more energy, which in turn prolongs the lifetime of the hosts. This may explain the crossing points in Fig. 12.

A longer BI also hurts the neighbor discovery time. As shown in Fig. 13, the neighbor discovery time will increase linearly as BI increases for both for $\mathrm{C}(98)$ and $\mathrm{E}(7 \times 14)$. The $\mathrm{E}(7 \times 14)$ scheme, which can tune its quorum intervals adaptively, has much shorter neighbor discovery time compared to $\mathrm{C}(98)$.

Fig. 14 shows the impact of BI on throughput. Longer beacon intervals do decrease throughputs. This is because it takes longer time for a host to wake up its neighboring PS hosts to help relay packets. The result shows that $\mathrm{E}(7 \times 14)$ slightly outperforms $\mathrm{C}(98)$ in terms of throughput due to its adaptivity, and the gain 


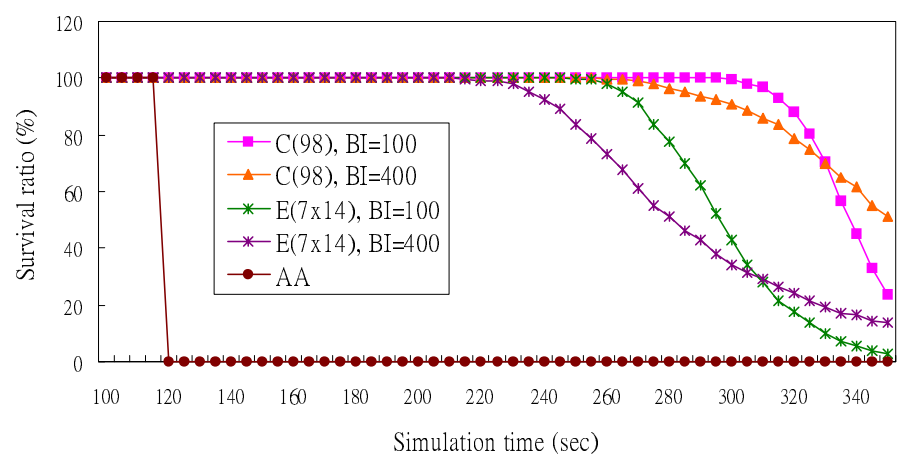

Figure 12: Survival ratio vs. beacon interval length (100 hosts, traffic load $=1$ route $/ \mathrm{sec}$, moving speed $=$ $0 \sim 20 \mathrm{~m} / \mathrm{sec}$ with mean $=10 \mathrm{~m} / \mathrm{sec})$.

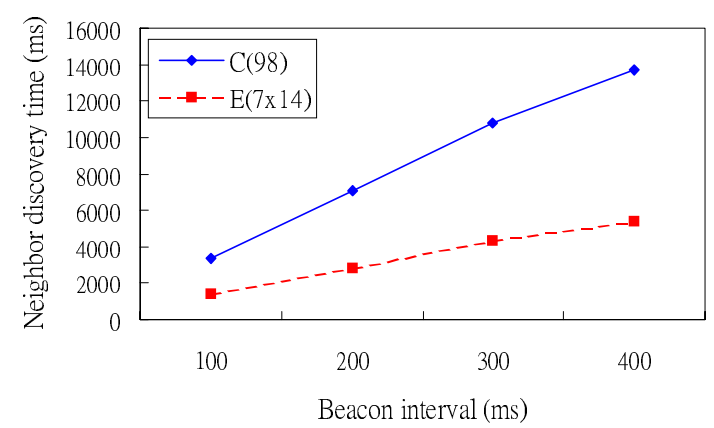

Figure 13: Neighbor discovery time vs. beacon interval length (100 hosts, traffic load $=1$ route/sec, moving speed $=0 \sim 20 \mathrm{~m} / \mathrm{sec}$ with mean $=10 \mathrm{~m} / \mathrm{sec}$ ).

will enlarge slightly as BI increases. However, $\mathrm{C}(98)$ outperfomrs $\mathrm{E}(7 \times 14)$ in terms of throughput $\times$ lifetime.

\subsubsection{Impact of Traffic Load}

Next, we observe the effect of traffic load. We vary the traffic load in the range of $1 \sim 4$ routes/sec in the simulations. Fig. 15 shows how traffic load decreases host survival ratios. Higher traffic loads do reduce host survival ratios of $\mathrm{C}(98)$ and $\mathrm{E}(7 \times 14)$, which is reasonable. On the contrary, the impact of traffic load on AA is insignificant because anyway hosts have to stay awake all the time. Overall, the proposed schemes still outperform the AA scheme in terms of survival ratio significantly. The effect of traffic load on throughput and accumulated throughput is shown in Fig. 16. The trend is similar to the

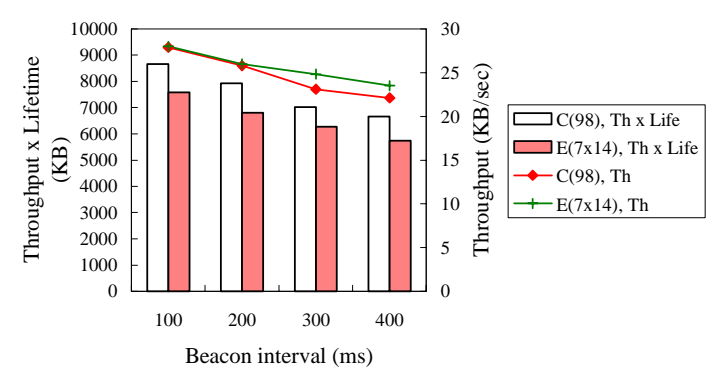

Figure 14: Throughput vs. beacon interval length $(100$ hosts, traffic load $=1$ route $/$ sec, moving speed $=$ $0 \sim 20 \mathrm{~m} / \mathrm{sec}$ with mean $=10 \mathrm{~m} / \mathrm{sec})$. 


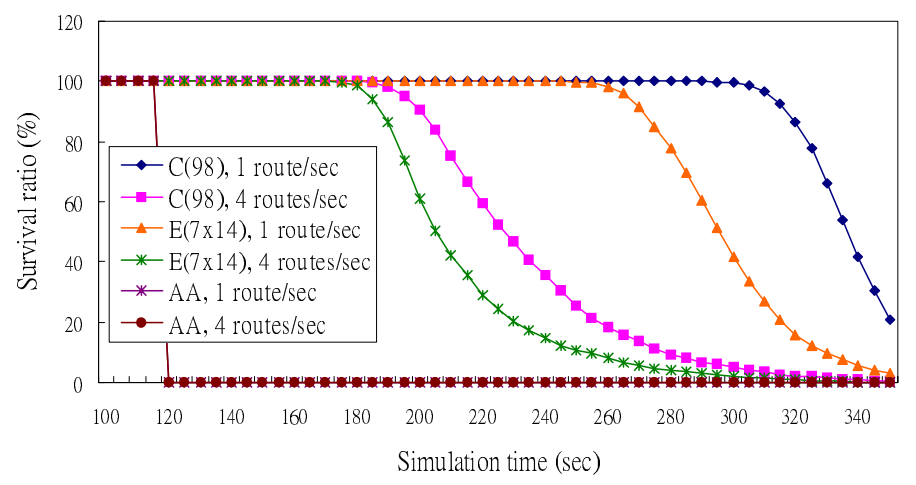

Figure 15: Survival ratio vs. traffic load (beacon interval $=100 \mathrm{~ms}, 100$ hosts, mobility $=020 \mathrm{~m} / \mathrm{sec}$ with mean $=10 \mathrm{~m} / \mathrm{sec})$.

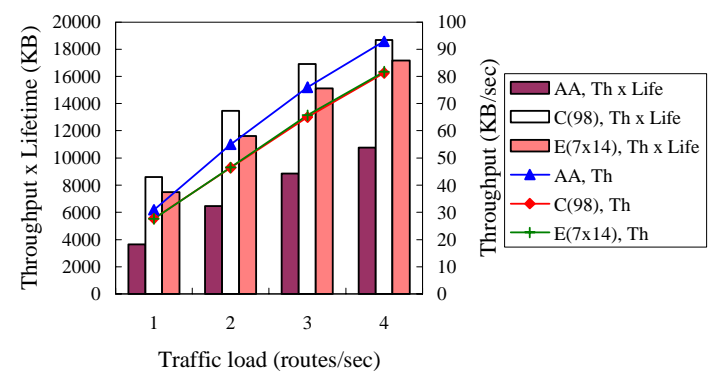

Figure 16: Throughput vs. traffic load (beacon interval $=100 \mathrm{~ms}, 100$ hosts, mobility $=0 \sim 20 \mathrm{~m} / \mathrm{sec}$ with mean $=10 \mathrm{~m} / \mathrm{sec})$.

earlier observation. Traffic load does not influence the neighbor discovery time much, so the related results are omitted.

\subsubsection{Impact of Host Density}

In this experiment, we vary the number of hosts in the range of 50 200. Since the network area is fixed, this parameter reflects the host density of the network. Fig. 17 shows that a higher host density will bring down the network lifetime. On the contrary, the AA scheme is almost unaffected. So a higher host density has a negative effect on survival ratio for our schemes. The reason can be explained as follows: As the network becomes denser, when a route request is issued, not only more hosts will help searching for routes, but also the broadcast cost per individual host will increase so as to wake up neighboring hosts (note that the traffic load remains unchanged in this case). In terms of survival ratio, $\mathrm{C}(98)$ outperforms $\mathrm{E}(7 \times 14)$, which in turn outperforms AA.

As Fig. 18 shows, a higher node density has a negative effect on throughput for quorum-based protocols, while it does not influence the AA scheme much. When the node density goes higher, broadcast cost will increase because of a higher wake-up cost. The effect is an increased number of collisions and a lower probability of route establishment. Consequently, the throughput goes down. According to Fig. 18, the throughput of the AA scheme is the highest, and the throughputs of the $\mathrm{E}(7 \times 14)$ and the $\mathrm{C}(98)$ schemes are very close. Again, when we consider "throughput $\times$ lifetime", our quorum-based protocols outperform 


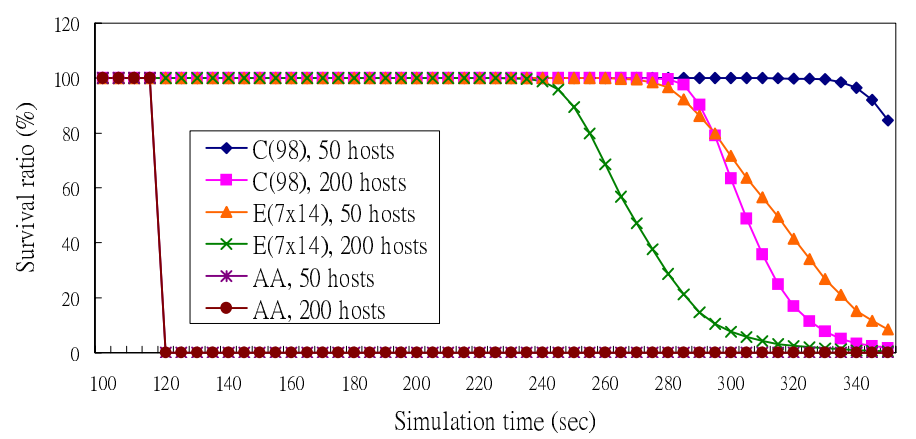

Figure 17: Survival ratio vs. host density (beacon interval $=100 \mathrm{~ms}$, traffic load 1 route $/ \mathrm{sec}$, mobility $=$ $0 \sim 20 \mathrm{~m} / \mathrm{sec}$ with mean $=10 \mathrm{~m} / \mathrm{sec})$.

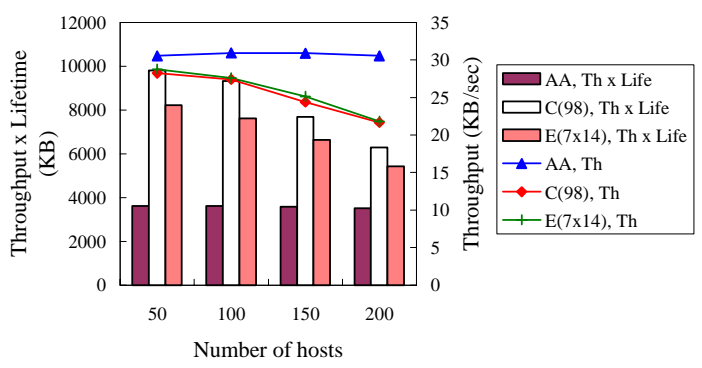

Figure 18: Throughput vs. node density (beacon interval $=100 \mathrm{~ms}$, traffic load 1 route $/ \mathrm{sec}$, mobility $=$ $0 \sim 20 \mathrm{~m} / \mathrm{sec}$ with mean $=10 \mathrm{~m} / \mathrm{sec})$.

the AA scheme significantly.

\section{Conclusions}

In this paper, we have addressed the asynchronous power mode management problem for an IEEE 802.11based MANET. We have correlated the problem to the concept of quorum systems and identified an important rotation closure property for quorum systems. We have proved that any quorum system satisfying the rotation closure property can be translated to an asynchronous power-saving protocol for MANETs. Under the rotation closure property, we have derived a quorum size lower bound for any quorum system. We have identified a group of optimal or near optimal quorum systems. Optimal or near optimal quorum systems are preferable because in a quorum-based power-saving protocol, the number of beacons sent and the ratio of a host remaining active are both proportional to the quorum size. We have shown that the grid quorum system [15], the torus quorum system [12], the cyclic quorum system [14], and the finite projective plane quorum system [15] are all optimal or near optimal quorum systems satisfying the rotation closure property. We have developed theorems to help identify good quorum systems satisfying the rotation closure property, such as quorum systems with only one member, which are very easy to maintain. We have further proposed a new e-torus quorum system, which can be translated to an adaptive power-saving protocol allowing hosts to dynamically tune to different quorum systems according to their mobility, so as to trade neighbor sensibility for power expenditure. Extensive simulation results have been presented to 
evaluate these protocols.

\section{References}

[1] B. Chen, K. Jamieson, H. Balakrishnan, and R. Morris. Span: An Energy-Efficient Coordination Algorithm for Topology Maintenance in Ad Hoc Wireless Networks. Proc. of the International Conference on Mobile Computing and Networking, pages 85-96, 2001.

[2] C. F. Chiasserini and R. R. Rao. A Distributed Power Management Policy for Wireless Ad Hoc Networks. IEEE Wireless Communication and Networking Conference, pages 1209-1213, 2000.

[3] C. J. Colbourn, J. H. Dinitz, and D. R. Stinson. Quorum Systems Constructed from Combinatorial Designs. Information and Computation, pages 160-173, 2001.

[4] C. J. Colbourn and E. J. H. Dinitz. The CRC Handbook of Combinatorial Designs. CRC Press, 1996.

[5] L. M. Feeney and M. Nilsson. Investigating the energy consumption of wireless network interface in an ad hoc networking environment. IEEE INFOCOM, pages 1548-1557, 2001.

[6] J. Gomez, A. T. Campbell, M. Naghshineh, and C. Bisdikian. A Distributed Contention Control Mechanism for Power Saving in random-access Ad-Hoc Wireless Local Area Networks. Proc. of IEEE International Workshop on Mobile Multimedia Communications, pages 114-123, 1999.

[7] J. C. Haartsen. The Bluetooth Radio System. IEEE Persinal Communications, pages 28-36, Feb 2000.

[8] L. Hu. Topology Control for Multihop Packet Radio Networks. IEEE Transactions on Communications, 41:1474-1481, Oct 1993.

[9] C. F. Huang, Y. C. Tseng, S. L. Wu, and J. P. Sheu. Increasing the Throughput of Multihop Packet Radio Networks with Power Adjustment. International Conference on Computer, Cummunication, and Networks, 2001.

[10] E.-S. Jung and N. H. Vaidya. An Energy Efficient MAC Protocol for Wireless LANs. INFOCOM 2002, 2002.

[11] LAN MAN Standards Committee of the IEEE Computer Society. IEEE Std 802.11-1999, Wireless LAN Medium Access Control (MAC) and Physical Layer (PHY) specifications. IEEE, 1999.

[12] S. D. Lang and L. J. Mao. A Torus Quorum Protocol for Distributed Mutual Exclusion. Proc. of the 10th Int'l Conf. on Parallel and Distributed Computing and Systems, pages 635-638, 1998. 
[13] J. R. Lorch and A. J. Smith. Software Strategies for Portable Computer Energy Management. IEEE Personal Communications, pages 60-73, Jun 1998.

[14] W. S. Luk and T. T. Wong. Two New Quorum Based Algorithms for Distributed Mutual Exclusion. Proc. of Int'l Conference on Distributed Computing Systems, pages 100-106, 1997.

[15] M. Maekawa. A $\sqrt{N}$ Algorithm for Mutual Exclusion in Decentralized Systems. ACM Trans. Comput. Syst., pages 145-159, 1985.

[16] C. E. Perkins and E. M. Belding-Royer. Ad-hoc on-demand distance vector routing. IEEE Workshop on Mobile Computing Systems and Applications, pages 90-100, 1999.

[17] R. Ramanathan and R. Rosales-Hain. Topology Control of Multihop Wireless Networks using Transmit Power Adjustment. IEEE INFOCOM, pages 404-413, 2000.

[18] J. H. Ryu and D. H. Cho. A New Routing Scheme Concerning Power-Saving in Mobile Ad-Hoc Networks. Proc. of IEEE International Conference on Communications, 3:1719-1722, 2000.

[19] J. H. Ryu, S. Song, and D. H. Cho. A Power-Saving Multicast Routing Scheme in 2-tier Hierarchical Mobile Ad-Hoc Networks. Proc. of IEEE Vehicular Technology Conference, 4:1974-1978, 2000.

[20] A. K. Salkintzis and C. Chamzas. An In-Band Power-Saving Protocol for Mobile Data Networks. IEEE Transactions on Communications, 46:1194-1205, Sep 1998.

[21] E. Shih, P. Bahl, and M. J. Sinclair. Wake on Wireless: An Event Driven Energy Saving Strategy for Battery Operated Devices. MOBICOM 2002, 2002.

[22] T. Simunic, H. Vikalo, P. Glynn, and G. D. Micheli. Energy Efficient Design of Portable Wireless Systems. Proc. of the International Symposium on Low Power Electronics and Design, pages 49-54, 2000.

[23] S. Singh and C. S. Raghavendra. Power Efficient MAC Protocol for Multihop Radio Networks. Proc. of IEEE International Personal, Indoor and Mobile Radio Communications Conference, pages 153-157, 1998.

[24] S. Singh, M. Woo, and C. S. Raghavendra. Power-Aware Routing in Mobile Ad Hoc Networks. Proc. of the International Conference on Mobile Computing and Networking, pages 181-190, 1998.

[25] Y. C. Tseng, C. S. Hsu, and T. Y. Hsieh. Power-Saving Protocols for IEEE 802.11-Based Multi-Hop Ad Hoc Networks. IEEE INFOCOM, 2002.

[26] R. Wattenhofer, L. Li, P. Bahl, and Y. M. Wang. Distributed Topology Control for Power Efficient Operation in Multihop Wireless Ad Hoc Networks. IEEE INFOCOM, pages 1388-1397, 2001. 
[27] H. Woesner, J. P. Ebert, M. Schlager, and A. Wolisz. Power-Saving Mechanisms in Emerging Standards for Wireless LANs: The MAC Level Perspective. IEEE Persinal Communications, pages 40-48, Jun 1998.

[28] S. L. Wu, Y. C. Tseng, and J. P. Sheu. Intelligent Medium Access for Mobile Ad Hoc Networks with BusyTones and Power Control. IEEE Journal on Selected Areas in Communications, 18:1647-1657, Sep 2000.

[29] Y. Xu, J. Heidemann, and D. Estrin. Geography-informed Energy Conservation for Ad Hoc Routing. Proc. of the International Conference on Mobile Computing and Networking, pages 70-84, 2001. 